\title{
Variable Lebesgue norm estimates for BMO functions II
}

\section{Mitsuo Izuki, Yoshihiro Sawano and Yohei Tsutsui}

\begin{tabular}{|c|l|}
\hline Citation & OCAMI Preprint Series \\
\hline Issue Date & 2012 \\
\hline Type & Preprint \\
\hline Textversion & Author \\
\hline Rights & For personal use only. No other uses without permission. \\
\hline Relation & $\begin{array}{l}\text { This is a pre-print of an article published in Analysis } \\
\text { Mathematica. The final authenticated version is available } \\
\text { online at: } \text { https://doi.org/10.1007/s10476-014-0304-6 } .\end{array}$ \\
\hline
\end{tabular}

From: Osaka City University Advanced Mathematical Institute http://www.sci.osaka-cu.ac.jp/OCAMI/publication/preprint/preprint.html 


\title{
Variable Lebesgue norm estimates for BMO functions II *
}

\author{
Mitsuo Izuki ${ }^{\dagger}$ Yoshihiro Sawano ${ }^{\ddagger}$ and Yohei Tsutsui ${ }^{\S}$
}

March 5, 2013

\begin{abstract}
The paper concerns characterization of BMO in terms of Banach function spaces. In particular, we are interested in characterizing BMO by using the variable Lebesgue norm.
\end{abstract}

Key words: BMO, variable exponent.

\section{Introduction}

We propose a property of the Hardy-Littlewood maximal operator $M$ here. For a measurable function $f: \mathbb{R}^{n} \rightarrow \mathbb{C}$, we define

$$
M f(x):=\sup _{r>0, y \in \mathbb{R}^{n} ; x \in y+(-r, r)^{n}} \frac{1}{(2 r)^{n}} \int_{y+[-r, r]^{n}}|f(z)| d z .
$$

To state our main results, we need to describe the Lebesgue space $L^{p(\cdot)}\left(\mathbb{R}^{n}\right)$ with variable exponent. For a measurable function $p(\cdot): \mathbb{R}^{n} \rightarrow[1, \infty)$, the Lebesgue space $L^{p(\cdot)}\left(\mathbb{R}^{n}\right)$ with variable exponent is defined to the set of all measurable functions $f$ on $\mathbb{R}^{n}$ for which the quantity

$$
\|f\|_{L^{p(\cdot)}}=\inf \left\{\lambda>0: \int_{\mathbb{R}^{n}}\left(\frac{|f(x)|}{\lambda}\right)^{p(x)} d x \leq 1\right\}
$$

is finite. We shall prove;

* Mathematics Subject Classifications 2010: 42B35

${ }^{\dagger}$ Department of Mathematics, Tokyo Denki University, Adachi-ku, Tokyo 120-8551, Japan. email: izuki@mail.dendai.ac.jp

${ }^{\ddagger}$ Department of Mathematics and Information Science, Tokyo Metropolitan University, 1-1 Minami-Ohsawa, Hachioji, Tokyo 192-0397, Japan. email: ysawano@tmu.ac.jp

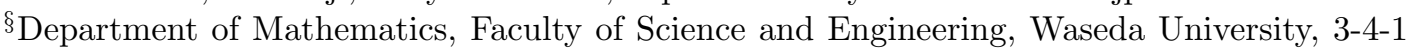
Okubo, Shinjuku, Tokyo 169-8555, Japan. email: y.tsutsui@kurenai.waseda.jp 
Theorem 1.1. Let $p(\cdot): \mathbb{R}^{n} \rightarrow[1, \infty)$ be a bounded function. Assume that the Hardy-Littlewood maximal operator $M$ is of weak type $(p(\cdot), p(\cdot))$, namely

$$
\sup _{\lambda>0} \lambda\left\|\chi_{\{M f(x)>\lambda\}}\right\|_{L^{p(\cdot)}} \leq C\|f\|_{L^{p(\cdot)}}
$$

holds for all measurable functions $f$. Then there exists a constant $0<\delta \leq 1$ such that

$$
\left\|f^{\delta}\right\|_{L^{p(\cdot)}} \leq C\left(\frac{1}{|Q|} \int_{Q} f(x) d x\right)^{\delta}\left\|\chi_{Q}\right\|_{L^{p(\cdot)}}
$$

for all positive measurable functions $f$ supported on a cube $Q$.

The space $\operatorname{BMO}\left(\mathbb{R}^{n}\right)$ is a famous space and it dates back to the paper of John and Nirenberg [12]. Theorem 1.1 enables us to characterize $\operatorname{BMO}\left(\mathbb{R}^{n}\right)$, the set of all locally integrable functions with bounded mean oscillation, by means of Banach function spaces.

In the whole paper we will use the following notation:

1. Given a measurable set $S \subset \mathbb{R}^{n}$, we denote the Lebesgue measure by $|S|$ and the characteristic function by $\chi_{S}$.

2. Given a measurable set $S \subset \mathbb{R}^{n}$ such that $0<|S|<\infty$ and a function $f$ on $\mathbb{R}^{n}$, we denote the mean value of $f$ on $S$ by $f_{S}$, namely $f_{S}:=\frac{1}{|S|} \int_{S} f(x) d x$.

3. A symbol $C$ always stands for a positive constant independent of the main parameters.

4. A cube $Q \subset \mathbb{R}^{n}$ is always assumed to be open and have sides parallel to the coordinate axes. Namely we can write

$$
Q=\prod_{\nu=1}^{n}\left(x_{\nu}-r / 2, x_{\nu}+r / 2\right)
$$

using a point $x=\left(x_{1}, x_{2}, \ldots, x_{n}\right) \in \mathbb{R}^{n}$ and a constant $r>0$.

5. The BMO space $\operatorname{BMO}\left(\mathbb{R}^{n}\right)$ consists of all $b \in L_{\text {loc }}^{1}\left(\mathbb{R}^{n}\right)$ such that

$$
\|b\|_{\mathrm{BMO}}:=\sup _{Q: \text { cube }} \frac{1}{|Q|} \int_{Q}\left|b(x)-b_{Q}\right| d x<\infty .
$$

We apply Theorem 1.1 and investigate the space $\operatorname{BMO}\left(\mathbb{R}^{n}\right)$ :

Theorem 1.2. Let $p(\cdot): \mathbb{R}^{n} \rightarrow[1, \infty)$ be a bounded function. Assume that the Hardy-Littlewood maximal operator $M$ is of weak type $(p(\cdot), p(\cdot))$. Then we have that for all $b \in \operatorname{BMO}\left(\mathbb{R}^{n}\right)$,

$$
C^{-1}\|b\|_{\mathrm{BMO}} \leq \sup _{Q: \text { cube }} \frac{1}{\left\|\chi_{Q}\right\|_{L^{p(\cdot)}}}\left\|\left(b-b_{Q}\right) \chi_{Q}\right\|_{L^{p(\cdot)}} \leq C\|b\|_{\mathrm{BMO}} .
$$


Theorem 1.2 gives an example of affirmative answers for the following problem:

Problem 1.3. Let $X$ be a subset of the set of all measurable functions on $\mathbb{R}^{n}$. Suppose that $X$ is a Banach function space eqquipped with a norm $\|\cdot\|_{X}$. We write

$$
\|b\|_{\mathrm{BMO}_{X}}:=\sup _{Q: \text { cube }} \frac{1}{\left\|\chi_{Q}\right\|_{X}}\left\|\left(b-b_{Q}\right) \chi_{Q}\right\|_{X}
$$

Can we say that there exists a constant $C>0$ such that

$$
C^{-1}\|b\|_{\mathrm{BMO}} \leq\|b\|_{\mathrm{BMO}_{X}} \leq C\|b\|_{\mathrm{BMO}}
$$

for all $b \in L_{\mathrm{loc}}^{1}\left(\mathbb{R}^{n}\right)$ ?

The first author and the second author proved the following results:

Theorem 1.4. Let $p(\cdot): \mathbb{R}^{n} \rightarrow[1, \infty)$ be a bounded variable exponent.

1. (Izuki [9]) If $p(\cdot)$ is an exponent for which $M$ is bounded on $L^{p(\cdot)}\left(\mathbb{R}^{n}\right)$, then we have that for all $b \in \mathrm{BMO}\left(\mathbb{R}^{n}\right)$,

$$
C^{-1}\|b\|_{\mathrm{BMO}} \leq \sup _{Q: \text { cube }} \frac{1}{\left\|\chi_{Q}\right\|_{L^{p(\cdot)}}}\left\|\left(b-b_{Q}\right) \chi_{Q}\right\|_{L^{p(\cdot)}} \leq C\|b\|_{\mathrm{BMO}}
$$

2. (Izuki-Sawano [10]) If $1 \leq p_{-}=\inf p(\cdot)$ and $p(\cdot) \in L H\left(\mathbb{R}^{n}\right)$, then equivalence (1.4) is also true.

We refer to Subsection 2.1 for the definition of $L H\left(\mathbb{R}^{n}\right)$.

Theorem 1.5 (Ho [8]). If the Hardy-Littlewood maximal operator $M$ is bounded on the associate space $X^{\prime}$, then we have that, for all $b \in \operatorname{BMO}\left(\mathbb{R}^{n}\right)$,

$$
C^{-1}\|b\|_{\mathrm{BMO}} \leq\|b\|_{\mathrm{BMO}_{X}} \leq C\|b\|_{\mathrm{BMO}} .
$$

We refer to the book [1] for the definition of Banach function spaces and we recall it in Subsection 2.2.

We note that Theorem 1.5 includes Theorem 1.4. However, Theorem 1.2 is an outrange of Theorem 1.5.

Here we organize the remaining part of this paper. We clarify some terminology in Section 2. In Section 3, we prove Theorems 1.1 and 1.2. In Section 4, we give an equivalence norm of $\mathrm{BMO}_{\mathrm{X}}$ under some condition on $X$. Section 5 contains another characterization of $\mathrm{BMO}\left(\mathbb{R}^{n}\right)$ by using the harmonic extension.

\section{Preliminaries}

\subsection{Lebesgue spaces with variable exponent}

Let $\Omega \subset \mathbb{R}^{n}$ be a measurable set such that $|\Omega|>0$. 
Definition 2.1. Given a measurable function $p(\cdot): \Omega \rightarrow[1, \infty]$, define the Lebesgue space with variable exponent

$$
L^{p(\cdot)}(\Omega):=\left\{f: \rho_{p}(f / \lambda)<\infty \text { for some } \lambda>0\right\},
$$

where

$$
\rho_{p}(f):=\int_{\{p(x)<\infty\}}|f(x)|^{p(x)} d x+\|f\|_{L^{\infty}(\{p(x)=\infty\})} .
$$

We additionally define

$$
\|f\|_{L^{p(\cdot)}}:=\|f\|_{L^{p(\cdot)(\Omega)}}=\inf \left\{\lambda>0: \rho_{p}(f / \lambda) \leq 1\right\} .
$$

The functional $\|\cdot\|_{L^{p(\cdot)}}$ is a norm of the space $L^{p(\cdot)}(\Omega)$. If a variable exponent $p(\cdot)$ equals to a constant, then $L^{p(\cdot)}(\Omega)$ is the usual Lebesgue space with norm coincidence.

\section{Definition 2.2.}

1. Given a variable exponent $p(\cdot): \Omega \rightarrow[1, \infty]$, we define

$$
p_{+}:=\|p\|_{L^{\infty}(\Omega)}, p_{-}:=\left\{\left(\frac{1}{p}\right)_{+}\right\}^{-1} .
$$

2. The set $\mathcal{P}(\Omega)$ consists of all variable exponents $p(\cdot)$ such that $1<p_{-} \leq p_{+}<$ $\infty$.

3. The set $\mathcal{B}(\Omega)$ consists of all $p(\cdot) \in \mathcal{P}(\Omega)$ such that the Hardy-Littlewood maximal operator $M$ is bounded on $L^{p(\cdot)}(\Omega)$.

4. A measurable function $r(\cdot): \Omega \rightarrow(0, \infty)$ is said to be globally log-Hölder continuous if the following two conditions are satisfied:

$$
\begin{gathered}
|r(x)-r(y)| \leq \frac{C}{-\log (|x-y|)} \quad(|x-y| \leq 1 / 2), \\
\left|r(x)-r_{\infty}\right| \leq \frac{C}{\log (e+|x|)} \quad(x \in \Omega),
\end{gathered}
$$

where $r_{\infty}$ is a real constant. The set $L H(\Omega)$ consists of all globally log-Hölder continuous functions.

The next proposition $([3,6])$ gives us a sufficient condition for the boundedness of the Hardy-Littlewood maximal operator.

Proposition 2.1. Suppose that a variable exponent $p(\cdot): \mathbb{R}^{n} \rightarrow[1, \infty]$ satisfies $1 \leq p_{-} \leq p_{+} \leq \infty$ and $1 / p(\cdot) \in L H\left(\mathbb{R}^{n}\right)$. Then $M$ is of weak type $(p(\cdot), p(\cdot))$, that is,

$$
\left\|\chi_{\{M f(x)>\lambda\}}\right\|_{L^{p(\cdot)}} \leq C \lambda^{-1}\|f\|_{L^{p(\cdot)}}
$$

holds for all $\lambda>0$ and all $f \in L^{p(\cdot)}\left(\mathbb{R}^{n}\right)$. Additionally if $1<p_{-}$, then $M$ is bounded on $L^{p(\cdot)}\left(\mathbb{R}^{n}\right)$, that is,

$$
\|M f\|_{L^{p(\cdot)}} \leq C\|f\|_{L^{p(\cdot)}} .
$$


We next state some equivalent conditions due to Diening [5]. Below $p^{\prime}(\cdot)$ means the conjugate exponent of $p(\cdot)$, that is, $1 / p(x)+1 / p^{\prime}(x)=1$ holds, and $\mathcal{Y}$ consists of all families of disjoint cubes.

Proposition 2.2. [5] Given a variable exponent $p(\cdot) \in \mathcal{P}\left(\mathbb{R}^{n}\right)$, the next four conditions are equivalent:

(D1) $p(\cdot) \in \mathcal{B}\left(\mathbb{R}^{n}\right)$.

(D2) $p^{\prime}(\cdot) \in \mathcal{B}\left(\mathbb{R}^{n}\right)$.

(D3) There exists a constant $q \in\left(1, p_{-}\right)$such that $p(\cdot) / q \in \mathcal{B}\left(\mathbb{R}^{n}\right)$.

(D4) For all $Y \in \mathcal{Y}$ and all $f \in L^{p(\cdot)}\left(\mathbb{R}^{n}\right)$, we have

$$
\left\|\sum_{Q \in Y}|f|_{Q} \chi_{Q}\right\|_{L^{p(\cdot)}} \leq C\|f\|_{L^{p(\cdot)} .}
$$

If we take an arbitrary cube $Q$ and put $Y=\{Q\}$ and $f=f \chi_{Q}$ in (D4) above, then we get a weaker condition

(A1) $|f|_{Q}\left\|\chi_{Q}\right\|_{L^{p(\cdot)}} \leq C\left\|f \chi_{Q}\right\|_{L^{p(\cdot)}}$ holds for all cubes $Q$ and all $f \in L^{p(\cdot)}\left(\mathbb{R}^{n}\right)$.

Condition (A1) is a necessary condition for the weak boundedness of $M$ on $L^{p(\cdot)}$ and equivalent to the following (A2) called the Muckenhoupt condition for a variable exponent $p(\cdot)$ :

(A2) $\sup _{Q: \text { cube }} \frac{1}{|Q|}\left\|\chi_{Q}\right\|_{L^{p(\cdot)}}\left\|\chi_{Q}\right\|_{L^{p^{\prime}(\cdot)}}<\infty$.

We will prove those facts in Lemmas 2.4 and 2.5 in the context of general Banach function spaces.

\subsection{Banach function spaces}

In this subsection we first recall of the definition and fundamental properties of Banach function spaces.

Definition 2.3. Let $\Omega \subset \mathbb{R}^{n}$ be a measurable subset with $|\Omega|>0$ and $\mathcal{M}(\Omega)$ the set of all measurable and complex-valued functions on $\Omega$. A linear space $X \subset \mathcal{M}(\Omega)$ is said to be a Banach function space if there exists a functional $\|\cdot\|_{X}: \mathcal{M}(\Omega) \rightarrow$ $[0, \infty]$ with the following conditions: Let $f, g, f_{j} \in \mathcal{M}(\Omega)(j=1,2, \ldots)$.

1. $f \in X$ if and only if $\|f\|_{X}<\infty$.

2. (Norm property):

(a) (Positivity): $\|f\|_{X} \geq 0$.

(b) (strict Positivity): $\|f\|_{X}=0$ if and only if $f=0$ a.e.. 
(c) (Homogeneity): $\|\lambda f\|_{X}=|\lambda| \cdot\|f\|_{X}$.

(d) (The triangle inequality): $\|f+g\|_{X} \leq\|f\|_{X}+\|g\|_{X}$.

3. (Symmetry): $\|f\|_{X}=\||f|\|_{X}$.

4. (Lattice property): If $0 \leq g \leq f$ a.e., then $\|g\|_{X} \leq\|f\|_{X}$.

5. (Fatou property): If $0 \leq f_{1} \leq f_{2} \leq \ldots$ and $\lim _{j \rightarrow \infty} f_{j}=f$, then

$$
\lim _{j \rightarrow \infty}\left\|f_{j}\right\|_{X}=\|f\|_{X}
$$

6. For all measurable sets $F$ with $|F|<\infty$, it follows $\left\|\chi_{F}\right\|_{X}<\infty$ and

$$
\int_{F}|f(x)| d x \leq C_{F}\|f\|_{X} \quad(f \in X)
$$

with the constant $C_{F}$ depending on $F$.

Definition 2.4. Let $X \subset \mathcal{M}(\Omega)$ be a Banach function space equipped with a norm $\|\cdot\|_{X}$. The associate space $X^{\prime}$ is defined by

$$
X^{\prime}:=\left\{f \in \mathcal{M}(\Omega):\|f\|_{X^{\prime}}<\infty\right\}
$$

where

$$
\|f\|_{X^{\prime}}:=\sup \left\{\left|\int_{\Omega} f(x) g(x) d x\right|:\|g\|_{X} \leq 1\right\}
$$

For example the Lebesgue space $L^{p(\cdot)}(\Omega)$ with variable exponent $p(\cdot): \Omega \rightarrow$ $[1, \infty]$ is a Banach function space and the associated space is $L^{p^{\prime}(\cdot)}(\Omega)$.

The following lemma consists of the generalized Hölder inequality and the norm equivalence for Banach function spaces.

Lemma 2.3. Let $X \subset \mathcal{M}(\Omega)$ be a Banach function space.

1. For all $f \in X$ and all $g \in X^{\prime}$, we have

$$
\int_{\Omega}|f(x) g(x)| d x \leq C\|f\|_{X}\|g\|_{X^{\prime}}
$$

2. For all $f \in X$ we have

$$
C^{-1}\|f\|_{X} \leq \sup \left\{\left|\int_{\Omega} f(x) g(x) d x\right|:\|g\|_{X^{\prime}} \leq 1\right\} \leq C\|f\|_{X} .
$$

In particular the space $\left(X^{\prime}\right)^{\prime}$ is equal to $X$.

As an application of the lemma above, we show the following equivalence.

Lemma 2.4. Let $X \subset \mathcal{M}\left(\mathbb{R}^{n}\right)$ be a Banach function space. Then the following two conditions are equivalent: 
(I)

$$
\sup _{Q: \text { cube }} \frac{1}{|Q|}\left\|\chi_{Q}\right\|_{X}\left\|\chi_{Q}\right\|_{X^{\prime}}<\infty
$$

(II) For all cubes $Q$ and all $f \in L_{\mathrm{loc}}^{1}\left(\mathbb{R}^{n}\right)$ we have

$$
|f|_{Q}\left\|\chi_{Q}\right\|_{X} \leq C\left\|f \chi_{Q}\right\|_{X}
$$

Proof. Take an open cube $Q$ and $f \in L_{\text {loc }}^{1}\left(\mathbb{R}^{n}\right)$ arbitarily. The implication (II) $\Rightarrow$ (I) is proved as follows;

$$
\begin{aligned}
\frac{1}{|Q|}\left\|\chi_{Q}\right\|_{X}\left\|\chi_{Q}\right\|_{X^{\prime}} & \leq \frac{C}{|Q|}\left\|\chi_{Q}\right\|_{X} \sup \left\{\int_{\mathbb{R}^{n}}|f(x)| \chi_{Q}(x) d x:\|f\|_{X} \leq 1\right\} \\
& =C \sup \left\{|f|_{Q}\left\|\chi_{Q}\right\|_{X}:\|f\|_{X} \leq 1\right\} \\
& \leq C \sup \left\{\left\|f \chi_{Q}\right\|_{X}:\|f\|_{X} \leq 1\right\} \\
& \leq C .
\end{aligned}
$$

On the other hand, from (I) and the Hölder inequality, (II) is verified;

$$
\begin{aligned}
|f|_{Q}\left\|\chi_{Q}\right\|_{X} & =\frac{1}{|Q|} \int_{Q}|f(y)| d y \cdot\left\|\chi_{Q}\right\|_{X} \\
& \leq C \cdot \frac{1}{|Q|}\left\|f \chi_{Q}\right\|_{X}\left\|\chi_{Q}\right\|_{X^{\prime}}\left\|\chi_{Q}\right\|_{X} \\
& \leq C\left\|f \chi_{Q}\right\|_{X} .
\end{aligned}
$$

Lemma 2.5. If the Hardy-Littlewood maximal operator $M$ is weak bounded on $X$, that is

$$
\left\|\chi_{\{M g>\lambda\}}\right\|_{X} \leq C \lambda^{-1}\|g\|_{X}
$$

holds for all $\lambda>0$ and all $g \in X$, then we have

$$
|f|_{Q}\left\|\chi_{Q}\right\|_{X} \leq C\left\|f \chi_{Q}\right\|_{X}
$$

for all cubes $Q$ and all $f \in L_{\mathrm{loc}}^{1}\left(\mathbb{R}^{n}\right)$.

Proof. Take a cube $Q$ and $f \in L_{\text {loc }}^{1}\left(\mathbb{R}^{n}\right)$ arbitrarily. If $|f|_{Q}=0$, then the conclusion is obviously true. Below we assume $|f|_{Q}>0$ and write $\lambda:=|f|_{Q} / 2$. Since $|f|_{Q} \chi_{Q}(x) \leq C M\left(f \chi_{Q}\right)(x)$ one has

$$
M\left(f \chi_{Q}\right)>\lambda \text { on } Q
$$

Thus, we get

$$
|f|_{Q}\left\|\chi_{Q}\right\|_{X} \leq|f|_{Q}\left\|\chi_{\left\{M\left(f \chi_{Q}\right)(x)>\lambda\right\}}\right\|_{X} \leq|f|_{Q} \cdot C \lambda^{-1}\left\|f \chi_{Q}\right\|_{X}=C\left\|f \chi_{Q}\right\|_{X} .
$$

This proves the lemma. 


\section{Proof of Theorems 1.1 and $\mathbf{1 . 2}$}

\subsection{Proof of Theorem 1.1}

We will use the next lemma in order to prove the theorem above.

\section{Lemma 3.1.}

(i) Let $r(\cdot): \mathbb{R}^{n} \rightarrow(0, \infty)$ be a bounded measurable function with $r_{+}=1$. It holds

$$
\|f+g\|_{L^{r(\cdot)}} \geq\|f\|_{L^{r(\cdot)}}+\|g\|_{L^{r(\cdot)}}
$$

for all positive measurable functions $f, g$.

(ii) Let $p(\cdot): \mathbb{R}^{n} \rightarrow(0, \infty)$ be a bounded measurable function with $p_{+} \geq 1$. It holds

$$
\|f+g\|_{L^{p(\cdot)}}^{p_{+}} \geq\|f\|_{L^{p(\cdot)}}^{p_{+}}+\|g\|_{L^{p(\cdot)}}^{p_{+}}
$$

for all positive measurable functions $f, g$.

Proof. We first prove (i). Note that

$$
((1-\theta) a+\theta b)^{r(x)} \geq(1-\theta) a^{r(x)}+\theta b^{r(x)},
$$

since $\phi_{x}(t):=t^{r(x)}$ is concave. Hence we have

$$
\begin{aligned}
& \int_{\mathbb{R}^{n}}\left(\frac{f(x)+g(x)}{\|f\|_{L^{r(\cdot)}}+\|g\|_{L^{r(\cdot)}}}\right)^{r(x)} d x \\
& =\int_{\mathbb{R}^{n}}\left(\frac{\|f\|_{L^{r(\cdot)}}}{\|f\|_{L^{r(\cdot)}}+\|g\|_{L^{r(\cdot)}}} \cdot \frac{f(x)}{\|f\|_{L^{r(\cdot)}}}+\frac{\|g\|_{L^{r(\cdot)}}}{\|f\|_{L^{r(\cdot)}}+\|g\|_{L^{r(\cdot)}}} \cdot \frac{g(x)}{\|g\|_{L^{r(\cdot)}}}\right)^{r(x)} d x \\
& \geq \int_{\mathbb{R}^{n}} \frac{\|f\|_{L^{r(\cdot)}}}{\|f\|_{L^{r(\cdot)}}+\|g\|_{L^{r(\cdot)}}}\left(\frac{f(x)}{\|f\|_{L^{r(\cdot)}}}\right)^{r(x)}+\frac{\|g\|_{L^{r(\cdot)}}}{\|f\|_{L^{r(\cdot)}}+\|g\|_{L^{r(\cdot)}}}\left(\frac{g(x)}{\|g\|_{L^{r(\cdot)}}}\right)^{r(x)} d x \\
& =1 .
\end{aligned}
$$

This is the desired result. Next we prove (ii) by applying (i) with $r(\cdot)=p(\cdot) / p_{+}$. Let $h$ be a positive measurable function. Observe that

$$
\|h\|_{L^{p(\cdot)}}^{p_{+}}=\left\|h^{p_{+}}\right\|_{L^{p(\cdot) / p_{+}}}
$$

and $(f+g)^{p_{+}} \geq f^{p_{+}}+g^{p_{+}}$. Therefore, we obtain

$$
\begin{aligned}
\|f\|_{L^{p(\cdot)}}^{p_{+}}+\|g\|_{L^{p(\cdot)}}^{p_{+}} & =\left\|f^{p_{+}}\right\|_{L^{p(\cdot) / p_{+}}}+\left\|g^{p_{+}}\right\|_{L^{p(\cdot) / p_{+}}} \\
& \leq\left\|f^{p_{+}}+g^{p_{+}}\right\|_{L^{p(\cdot) / p_{+}}} \\
& \leq\left\|(f+g)^{p_{+}}\right\|_{L^{p \cdot \cdot) / p_{+}}} \\
& =\|f+g\|_{L^{p(\cdot)}}^{p_{+}} .
\end{aligned}
$$

Thus, the proof is complete.

Proof of Theorem 1.1. 
1. To prove Theorem 1.1, we invoke the following preliminary observations: We set $Q_{0,0}:=E_{0}:=Q=x_{Q}+[0, r]^{n}$. By a dyadic cube of $Q$ we mean the set $\left\{x_{Q}+2^{-m} z+2^{-m} w: w \in[0, r]^{n}, m=0,1,2, \cdots, z \in\left\{0,1,2, \cdots, 2^{m}-1\right\}\right\}$.

First of all, we let

$$
E_{k}=\left\{x \in Q: 2^{(n+1)(k-1)} f_{Q} \leq M^{d, Q} f(x)\right\},
$$

where $M^{d, Q}$ denotes the dyadic maximal operator with respect to $Q$, namely,

$$
M^{d, Q} f(x)=\sup \left\{\chi_{R}(x)|f|_{R}: R: \text { dyadic cubes of } Q\right\} .
$$

By the definition of the dyadic maximal operator $M^{d, Q}$, we obtain a family of non-overlapping cubes $\left\{Q_{k, l}\right\}_{l \in L_{k}}$ such that

$$
\sum_{l \in L_{k}} Q_{k, l}=E_{k}
$$

and that

$$
\frac{1}{\left|Q_{k, l}\right|} \int_{Q_{k, l}} f(y) d y>2^{(n+1)(k-1)} f_{Q} \geq \frac{1}{2^{n}\left|Q_{k, l}\right|} \int_{Q_{k, l}} f(y) d y .
$$

Note that $E_{0} \cup\left(\bigcup_{k=1}^{\infty} E_{k+1} \backslash E_{k}\right)$ differs from $Q$ by a set of measure zero. Hence,

$$
\begin{aligned}
f(x) & \leq M^{d, Q} f(x) \\
& =M^{d, Q} f(x) \chi_{Q_{0} \backslash E_{1}}(x)+\sum_{k=1}^{\infty} M^{d, Q} f(x) \chi_{E_{k} \backslash E_{k+1}}(x) \\
& \leq 2^{n+1} f_{Q} \chi_{E_{1}}(x)+\sum_{k=1}^{\infty} 2^{(n+1)(k+1)} f_{Q} \chi_{E_{k} \backslash E_{k+1}}(x) \\
& \leq 2^{n+1} \sum_{k=1}^{\infty} 2^{(n+1) k} f_{Q} \chi_{E_{k}}(x)
\end{aligned}
$$

as we did in [15]. Here for the last inequality, we have used the fact that $E_{0} \subset E_{1}$.

2. About the structure of $E_{k}$, we can prove

$$
\left|E_{k+1} \cap Q_{k, l}\right| \leq \frac{1}{2}\left|Q_{k, l}\right|
$$

by way of (3.1) and the decomposition

$$
E_{k+1} \cap Q_{k, l}=\bigcup_{l^{\prime} \in L_{k, l}} Q_{k+1, l^{\prime}}
$$


with $L_{l, k} \subset L_{k}$. See $[15$, p.3688] for details. Hence we have

$$
\left|E_{k+1} \cap Q_{k, l}\right| \leq \frac{1}{2}\left|Q_{k, l}\right| .
$$

By virtue of the weak boundedness of $M$, we have

$$
\begin{aligned}
\frac{1}{2}\left\|\chi_{E_{k}}\right\|_{L^{p(\cdot)}} & =\frac{1}{2}\left\|\chi_{\left\{\sum_{l \in L_{k}}\left(2 \chi_{E_{k} \backslash E_{k+1}}\right)_{Q_{k, l}} \chi_{Q_{k, l}}>1\right\}}\right\|_{L^{p(\cdot)}} \\
& \leq \frac{1}{2}\left\|\chi_{\left\{M\left(2 \chi_{E_{k} \backslash E_{k+1}}\right)>1\right\}}\right\|_{L^{p(\cdot)}} \\
& \leq C\left\|\chi_{E_{k} \backslash E_{k+1}}\right\|_{L^{p(\cdot)}} .
\end{aligned}
$$

Consequently,

$$
\left\|\chi_{E_{k}}\right\|_{L^{p(\cdot)}} \leq 2 C\left\|\chi_{E_{k} \backslash E_{k+1}}\right\|_{L^{p(\cdot)}} .
$$

Hence by using Lemma 3.1, we have

$$
\|f+g\|_{L^{p(\cdot)}}^{p_{+}} \geq\|f\|_{L^{p(\cdot)}}^{p_{+}}+\|g\|_{L^{p(\cdot)}}^{p_{+}}
$$

which holds for all positive measurable functions $f, g$. In particular,

$$
\left\|\chi_{E_{k}}\right\|_{L^{p(\cdot)}}^{p_{+}} \geq\left\|\chi_{E_{k+1}}\right\|_{L^{p(\cdot)}}^{p_{+}}+\left\|\chi_{E_{k} \backslash E_{k+1}}\right\|_{L^{p(\cdot)}}^{p_{+}} .
$$

Thus, combining (3.3) and (3.4), we obtain

$$
\left\|\chi_{E_{k}}\right\|_{L^{p(\cdot)}}^{p_{+}} \geq\left\|\chi_{E_{k+1}}\right\|_{L^{p(\cdot)}}^{p_{+}}+\left(\frac{1}{2 C}\left\|\chi_{E_{k}}\right\|_{L^{p(\cdot)}}\right)^{p_{+}}
$$

we conclude that

$$
\left\|\chi_{E_{k+1}}\right\|_{L^{p(\cdot)}} \leq\left(1-\left(\frac{1}{2 C}\right)^{p_{+}}\right)^{1 / p_{+}}\left\|\chi_{E_{k}}\right\|_{L^{p(\cdot)}} .
$$

Thus, we have

$$
\left\|\chi_{E_{k}}\right\|_{L^{p(\cdot)}} \leq\left(1-\left(\frac{1}{2 C}\right)^{p_{+}}\right)^{1 / p_{+}}\left\|\chi_{Q}\right\|_{L^{p(\cdot)}}
$$

3. If we combine (3.2) and (3.5), then we take a positive constant $\delta \leq 1$ so that

$$
2^{(n+1) \delta / p_{-}}\left(1-\left(\frac{1}{2 C}\right)^{p_{+}}\right)^{1 /\left(p_{+} p_{-}\right)}<1
$$

and obtain

$$
\begin{aligned}
\left\|f^{\delta}\right\|_{L^{p(\cdot)}}^{1 / p_{-}} & \leq\left\|\sum_{k=1}^{\infty} 2^{(n+1) k \delta}\left(f_{Q}\right)^{\delta} \chi_{E_{k}}\right\|_{L^{p(\cdot)}}^{1 / p_{-}} \\
& \leq \sum_{k=1}^{\infty} 2^{(n+1) k \delta / p_{-}}\left\|\left(f_{Q}\right)^{\delta} \chi_{E_{k}}\right\|_{L^{p(\cdot)}}^{1 / p_{-}} \\
& \leq \sum_{k=1}^{\infty} 2^{(n+1) k \delta / p_{-}}\left(f_{Q}\right)^{\delta / p_{-}}\left(1-\left(\frac{1}{2 C}\right)^{p_{+}}\right)^{k /\left(p_{+} p_{-}\right)}\left\|\chi_{Q}\right\|_{L^{p(\cdot)}}^{1 / p_{-}} \\
& \leq C\left(f_{Q}\right)^{\delta / p_{-}}\left\|\chi_{Q}\right\|_{L^{p(\cdot)}}^{1 / p_{-}} .
\end{aligned}
$$

This is the desired result. 


\subsection{Proof of Theorem $\mathbf{1 . 2}$}

As an application of Theorem 1.1, we prove Theorem 1.2.

Proof. We give the proof based on [10]. Take a cube $Q$ and $b \in \operatorname{BMO}\left(\mathbb{R}^{n}\right)$ arbitrarily. By virtue of Lemma 2.5 we see that

$$
|g|_{Q}\left\|\chi_{Q}\right\|_{L^{p(\cdot)}} \leq C\left\|g \chi_{Q}\right\|_{L^{p(\cdot)}}
$$

holds for all $g \in L_{\text {loc }}^{1}\left(\mathbb{R}^{n}\right)$. By putting $g:=b-b_{Q}$, we can immediately get the left hand side inequality of (1.3). Applying Theorem 1.1 with $f:=\left|b-b_{Q}\right|^{1 / \delta} \chi_{Q}$ with $\delta \in(0,1]$, the other implication is verified as follows;

$$
\begin{aligned}
\left\|\left(b-b_{Q}\right) \chi_{Q}\right\|_{L^{p(\cdot)}} & \leq C\left(\frac{1}{|Q|} \int_{Q}\left|b(x)-b_{Q}\right|^{1 / \delta} d x\right)^{\delta}\left\|\chi_{Q}\right\|_{L^{p(\cdot)}} \\
& \leq C\|b\|_{\mathrm{BMO}}\left\|\chi_{Q}\right\|_{L^{p(\cdot)}} .
\end{aligned}
$$

Thus, Theorem 1.2 is proved.

\section{Another characterization of $\mathrm{BMO}_{\mathrm{X}}\left(\mathbb{R}^{\mathrm{n}}\right)$}

We know several equivalence norms of $\operatorname{BMO}\left(\mathbb{R}^{n}\right)$. It is well-known that

$$
\sup _{Q: \text { cube }} \inf _{c \in \mathbb{C}} \frac{1}{\left\|\chi_{Q}\right\|_{L^{p}}}\left\|(b-c) \chi_{Q}\right\|_{L^{p}}
$$

with $p \in[1, \infty)$ is equivalent to the original one $\|b\|_{\text {BMO }}$. In [14] Muckenhoupt and Wheeden proved that, for the weight $w$ belonging to Muckenhoupt class $A_{\infty}$,

$$
\|b\|_{\mathrm{BMO}(w)}=\sup _{Q} \frac{1}{w(Q)} \int_{Q}\left|b(x)-b_{Q ; w}\right| w(x) d x
$$

where $w(Q):=\int_{Q} w(x) d x$ and $b_{Q ; w}:=\frac{1}{w(Q)} \int_{Q} b(x) w(x) d x$, is also equivalent

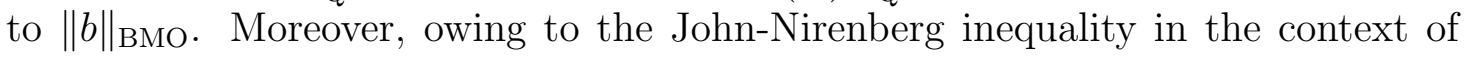
non-doubling measures by Mateu, Mattila, Nicolau and Orobitg [13], we see that for the same weight $w$ above

$$
C^{-1} \sup _{Q}\left\langle b-b_{Q}\right\rangle_{\exp L(Q ; w)} \leq\|b\|_{\mathrm{BMO}} \leq C \sup _{Q}\left\langle b-b_{Q}\right\rangle_{\exp L(Q ; w)},
$$

where

$$
\langle f\rangle_{\exp L(Q ; w)}=\inf \left\{\lambda>0:\left(\exp \left(\frac{|f|}{\lambda}\right)-1\right)_{Q ; w} \leq 1\right\} .
$$

In this subsection, we establish the same equivalence with " $\inf _{c \in \mathbb{C}}$ " instead of the average $b_{Q}$ in the context of Banach function spaces under a condition. 
Theorem 4.1. If Banach function space $X$ satisfies

$$
\sup _{Q: \text { cube }} \frac{1}{|Q|}\left\|\chi_{Q}\right\|_{X}\left\|\chi_{Q}\right\|_{X^{\prime}}<\infty
$$

then it follows

$$
C\|b\|_{\mathrm{BMO}_{X}} \leq \sup _{Q: \text { cube }} \inf _{c \in \mathbb{C}} \frac{1}{\left\|\chi_{Q}\right\|_{X}}\left\|(b-c) \chi_{Q}\right\|_{X} \leq\|b\|_{\mathrm{BMO}_{X}}
$$

for all measurable functions $b$.

Proof. The right-hand side inequality is obvious. Applying Lemma 2.3, we can verify the left-hand one as follows; for a cube $Q$ and $c \in \mathbb{C}$,

$$
\begin{aligned}
\frac{1}{\left\|\chi_{Q}\right\|_{X}}\left\|\left(b-b_{Q}\right) \chi_{Q}\right\|_{X} & \leq \frac{1}{\left\|\chi_{Q}\right\|_{X}}\left\{\left\|(b-c) \chi_{Q}\right\|_{X}+\left\|\left(c-b_{Q}\right) \chi_{Q}\right\|_{X}\right\} \\
& =\frac{1}{\left\|\chi_{Q}\right\|_{X}}\left\|(b-c) \chi_{Q}\right\|_{X}+\left|c-b_{Q}\right| \\
& \leq \frac{1}{\left\|\chi_{Q}\right\|_{X}}\left\|(b-c) \chi_{Q}\right\|_{X}+\frac{1}{|Q|} \int_{Q}|b-c| d x \\
& \leq \frac{1}{\left\|\chi_{Q}\right\|_{X}}\left\|(b-c) \chi_{Q}\right\|_{X}+C \frac{1}{|Q|}\left\|(b-c) \chi_{Q}\right\|_{X}\left\|_{\chi_{Q}}\right\|_{X^{\prime}} \\
& \leq \frac{C}{\left\|\chi_{Q}\right\|_{X}}\left\|(b-c) \chi_{Q}\right\|_{X} .
\end{aligned}
$$

Remark 4.1 .

1. For example, $X=\exp L\left(\mathbb{R}^{n}\right)$ satisfies the condtion in Theorem 4.1, where $\exp L\left(\mathbb{R}^{n}\right)$ denotes the set of all functions $f$ such that

$$
\|f\|_{\exp L}=\inf \left\{\lambda>0: \int_{\mathbb{R}^{n}}\left\{\exp \left(\frac{|f(x)|}{\lambda}\right)-1\right\} d x \leq 1\right\}<\infty .
$$

In fact, it holds that $\left\|\chi_{Q}\right\|_{\exp L}=\frac{1}{\log (1+1 /|Q|)}$ and that

$$
\left\|\chi_{Q}\right\|_{(\exp L)^{\prime}} \leq c|Q| \log (1+1 /|Q|) .
$$

2. The same argument with fundamental fact $|b|_{Q} \leq 2\langle b\rangle_{\exp L(Q)}$, see [16] for the proof, yields the equivalence

$$
\sup _{Q}\left\langle b-b_{Q}\right\rangle_{\exp L(Q)} \sim \sup _{Q} \inf _{c \in \mathbb{C}}\langle b-c\rangle_{\exp L(Q)},
$$

with $\langle f\rangle_{\exp L(Q)}=\langle f\rangle_{\exp L(Q ; 1)}$.

Combining Theorems 1.2 and 4.1, we get another equivalence norm of $\operatorname{BMO}\left(\mathbb{R}^{n}\right)$ by means of variable exponent spaces.

Corollary 4.2. Let $p(\cdot): \mathbb{R}^{n} \rightarrow[1, \infty)$ be a bounded function. Assume that $M$ is of weak type $(p(\cdot), p(\cdot))$. Then we have that for $b \in \operatorname{BMO}\left(\mathbb{R}^{n}\right)$,

$$
C^{-1}\|b\|_{\text {BMO }} \leq \sup _{Q: \text { cube }} \inf _{c \in \mathbb{C}} \frac{1}{\left\|\chi_{Q}\right\|_{L^{p(\cdot)}}}\left\|(b-c) \chi_{Q}\right\|_{L^{p(\cdot)}} \leq C\|b\|_{\text {BMO }}
$$




\section{A characterization by way of harmonic exten- sion}

Let $1 \leq p<\infty$ be a constant. The $\operatorname{BMO}\left(\mathbb{R}^{n}\right)$ norm $\|b\|_{\text {BMO }}$ is equivalent to

$$
\sup _{(x, t) \in \mathbb{R}^{n} \times(0, \infty)}\left(\int_{\mathbb{R}^{n}}|b(y)-u(y, t)|^{p} P_{t}(x-y) d y\right)^{1 / p},
$$

where $P_{t}$ is the Poisson kernel given by

$$
P_{t}(x):=\frac{1}{\left(|x|^{2}+t^{2}\right)^{\frac{n+1}{2}}} \quad\left(x \in \mathbb{R}^{n}, t>0\right)
$$

and $u(x, t)=\left(b * P_{t}\right)(x)$.

Remark 5.1. Chen-Lau [2] proved the equivalence replacing $P_{t}$ by a more general function. Here for the sake of convenience, we include the proof and provide an alternative interpretation. By virtue of [4, Theorem 3.2], we know that

$$
\sup _{(x, t) \in \mathbb{R}^{n} \times(0, \infty)}\left(\frac{1}{t^{n}} \int_{B(x, t)}|b(y)-u(y, t)|^{p} d y\right)^{1 / p}
$$

is an equivalent norm for $b \in \operatorname{BMO}\left(\mathbb{R}^{n}\right)$. That is,

$$
\sup _{(x, t) \in \mathbb{R}^{n} \times(0, \infty)}\left(\frac{1}{t^{n}} \int_{B(x, t)}|b(y)-u(y, t)|^{p} d y\right)^{1 / p} \sim\|b\|_{\mathrm{BMO}}
$$

By the definition of poisson kernel, we have

$$
\begin{aligned}
& \sup _{(x, t) \in \mathbb{R}^{n} \times(0, \infty)}\left(\frac{1}{t^{n}} \int_{B(x, t)}|b(y)-u(y, t)|^{p} d y\right)^{1 / p} \\
& \leq C \sup _{(x, t) \in \mathbb{R}^{n} \times(0, \infty)}\left(\int_{\mathbb{R}^{n}}|b(y)-u(y, t)|^{p} P_{t}(x-y) d y\right)^{1 / p} .
\end{aligned}
$$

Meanwhile,

$$
\begin{aligned}
& \sup _{(x, t) \in \mathbb{R}^{n} \times(0, \infty)}\left(\int_{\mathbb{R}^{n}}|b(y)-u(y, t)|^{p} P_{t}(x-y) d y\right)^{1 / p} \\
& \leq \sup _{(x, t) \in \mathbb{R}^{n} \times(0, \infty)} \sum_{k \in \mathbb{Z}^{n}}\left(\int_{B(x+k t, 2 n t)}|b(y)-u(y, t)|^{p} P_{t}(x-y) d y\right)^{1 / p} \\
& \leq C \sup _{(x, t) \in \mathbb{R}^{n} \times(0, \infty)} \sum_{k \in \mathbb{Z}^{n}}(1+|k|)^{-n-1}\left(\int_{B(x+k t, 2 n t)}|b(y)-u(y, t)|^{p} d y\right)^{1 / p} \\
& \leq C \sup _{(x, t) \in \mathbb{R}^{n} \times(0, \infty)}\left(\frac{1}{t^{n}} \int_{B(x, t)}|b(y)-u(y, t)|^{p} d y\right)^{1 / p} \sim\|b\|_{\mathrm{BMO}} .
\end{aligned}
$$


We can generalize the result in view of variable exponent.

Theorem 5.1. Let $p(\cdot): \mathbb{R}^{n} \rightarrow[1, \infty)$ be a variable exponent such that

$$
1 \leq p_{-} \leq p_{+}<\infty
$$

Then we have that for all $b \in \mathrm{BMO}\left(\mathbb{R}^{n}\right)$,

$$
C^{-1}\|b\|_{\mathrm{BMO}} \leq\|b\|_{\mathrm{BMO}_{p(\cdot)}} \leq C\|b\|_{\mathrm{BMO}}
$$

where

$$
\begin{aligned}
& \|b\|_{\mathrm{BMO}_{p(\cdot)}} \\
& :=\inf \left\{\lambda>0: \sup _{(x, t) \in \mathbb{R}^{n} \times(0, \infty)} \int_{\mathbb{R}^{n}}\left|\frac{b(y)-u(x, t)}{\lambda}\right|^{p(y)} P_{t}(x-y) d y \leq 1\right\} .
\end{aligned}
$$

Proof. As we have mentioned, the result is known when $p(\cdot)$ is a constant. Since

$$
u^{p(\cdot)} \leq u^{p_{-}}+u^{p_{+}} \quad \text { for all } u>0,
$$

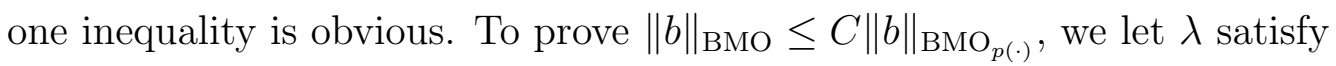

$$
\int_{\mathbb{R}^{n}}\left|\frac{b(y)-u(x, t)}{\lambda}\right|^{p(y)} P_{t}(x-y) d y \leq 1
$$

for all $(x, t) \in \mathbb{R}^{n} \times(0, \infty)$. Then

$$
\int_{\mathbb{R}^{n}}\left(\frac{1}{2}\left|\frac{b(y)-u(x, t)}{\lambda}\right|+\frac{1}{2}\right)^{p(y)} P_{t}(x-y) d y \leq 1 .
$$

Since

$$
\frac{t}{2} \leq\left(\frac{t+1}{2}\right)^{p(x)}
$$

it follows that

$$
\int_{\mathbb{R}^{n}}\left|\frac{b(y)-u(x, t)}{2 \lambda}\right|^{p(y)} P_{t}(x-y) d y \leq 1 .
$$

Thus, the proof is complete.

\section{Acknowledgements}

Mitsuo Izuki was partially supported by Grand-in-Aid for Scientific Research (C), No. 24540185, for Japan Society for the Promotion of Science. Yoshihiro Sawano was supported by Grant-in-Aid for Young Scientists (B) No. 24740085 Japan Society for the Promotion of Science. Yohei Tsutsui was supported by Research Fellowships of Japan Society for the Promotion of Science. Yohei Tsutsui was also partially supported by the JSPS Strategic Young Researcher Overseas Visits Program for Accelerating Brain Circulation "Deepening and Evolution of Mathematics and Physics, Buildingof International Network Hubbased on OCAMI". 


\section{References}

[1] C. Bennett and R. Sharpley, Interpolation of Operators, Academic Press, Boston, San Diego, New York, 1988.

[2] Y. Z. Chen and K. S. Lau, On an equivalent class of norms for BMO, J. Austral. Math. Soc. (Series A) 46 (1989), 289-295.

[3] D. Cruz-Uribe, L. Diening and A. Fiorenza, A new proof of the boundedness of maximal operators on variable Lebesgue spaces, Bull. Unione mat. Ital. (9) 2 (1) (2009), 151-173.

[4] D. Deng, X. T. Duong and L. Yan, A characterization of the MorreyCampanato spaces, Math. Z. 250 (2005), no. 3, 641-655.

[5] L. Diening, Maximal functions on Musielak-Orlicz spaces and generalized Lebesgue spaces, Bull. Sci. Math. 129 (2005), 657-700.

[6] L. Diening, P. Harjulehto, P. Hästö, Y. Mizuta and T. Shimomura, Maximal functions in variable exponent spaces: limiting cases of the exponent, Ann. Acad. Sci. Fenn. Math. 34 (2009), 503-522.

[7] L. Diening, P. Harjulehto, P. Hästö and M. Růžička, Lebesgue and Sobolev Spaces with Variable Exponents, Lecture Notes in Math. 2017, SpringerVerlag, Berlin, 2011.

[8] K.-P. Ho, Atomic decomposition of Hardy spaces and characterization of BMO via Banach function spaces, Anal. Math. 38 (2012), 173-185.

[9] M. Izuki, Boundedness of commutators on Herz spaces with variable exponent, Rend. Circ. Mat. Palermo, 59 (2010), 199-213.

[10] M. IzUKI AND Y. SAWANO, Variable Lebesgue norm estimates for BMO functions, Czechoslovak Mathematical Journal 62 (137) (2012), 717-727.

[11] O. Kováčik and J. Rákosník, On spaces $L^{p(x)}$ and $W^{k, p(x)}$, Czechoslovak Math. 41 (116) (1991), 592-618.

[12] J. John and L. Nirenberg, On function of bounded mean oscillation, Comm. Pure and Appl. Math. 14 (1961), 415-426.

[13] J. Mateu, P. Mattila, A. Nicolau and J. Orobitg, BMO for nondoubling measures, Duke Math. 102, 533-565, (2000).

[14] B. Muckenhoupt and R. Wheeden, Weighted bounded mean oscillation and the Hilbert transform, Studia Math. 54, (1975/76), 221-237.

[15] E. Nakai and Y. Sawano, Hardy spaces with variable exponents and generalized Campanato spaces, J. Funct. Anal. 262 (2012) 3665-3748.

[16] Y. Tsutsui, $A_{\infty}$ constant between BMO and weighted BMO. Submitted. 